This article was downloaded by: [Erasmus University]

On: 14 August 2015, At: 06: 28

Publisher: Routledge

Informa Ltd Registered in England and Wales Registered Number: 1072954 Registered office: 5 Howick Place, London, SW1P IWG

$+\frac{1}{2}$

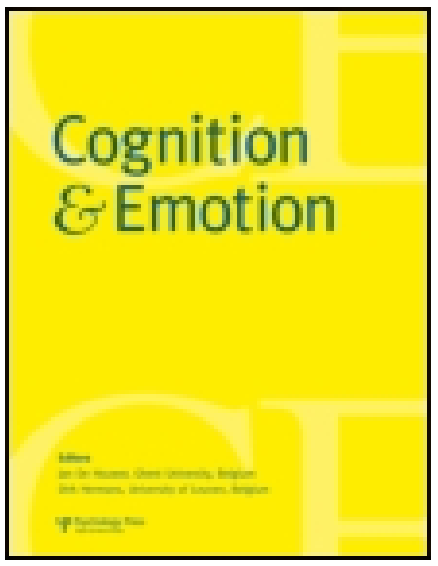

\title{
Cognition and Emotion
}

Publication details, including instructions for authors and subscription information:

http:/ / www.tandfonline.com/loi/ pcem20

\section{Emotional and non-emotional memories are suppressible under direct suppression instructions}

\author{
Kevin van Schie ${ }^{a}$, Elke Geraerts ${ }^{a} \&$ Michael C. Anderson ${ }^{b}$ \\ a Institute of Psychology, Erasmus University Rotterdam, Rotterdam , The \\ Netherlands \\ ${ }^{b}$ MRC Cognition and Brain Sciences Unit, Cambridge, UK \\ Published online: 07 Feb 2013.
}

To cite this article: Kevin van Schie, Elke Geraerts \& Michael C. Anderson (2013) Emotional and nonemotional memories are suppressible under direct suppression instructions, Cognition and Emotion, 27:6, 1122-1131, DOI: 10.1080/ 02699931.2013.765387

To link to this article: http:// dx.doi.org/ 10.1080/ 02699931.2013.765387

\section{PLEASE SCROLL DOWN FOR ARTICLE}

Taylor \& Francis makes every effort to ensure the accuracy of all the information (the "Content") contained in the publications on our platform. However, Taylor \& Francis, our agents, and our licensors make no representations or warranties whatsoever as to the accuracy, completeness, or suitability for any purpose of the Content. Any opinions and views expressed in this publication are the opinions and views of the authors, and are not the views of or endorsed by Taylor \& Francis. The accuracy of the Content should not be relied upon and should be independently verified with primary sources of information. Taylor and Francis shall not be liable for any losses, actions, claims, proceedings, demands, costs, expenses, damages, and other liabilities whatsoever or howsoever caused arising directly or indirectly in connection with, in relation to or arising out of the use of the Content.

This article may be used for research, teaching, and private study purposes. Any substantial or systematic reproduction, redistribution, reselling, loan, sub-licensing, systematic supply, or distribution in any form to anyone is expressly forbidden. Terms \& Conditions of access and use can be found at http://www.tandfonline.com/page/terms-and-conditions 


\title{
BRIEF REPORT
}

\section{Emotional and non-emotional memories are suppressible under direct suppression instructions}

\author{
Kevin van Schie ${ }^{1}$, Elke Geraerts ${ }^{1}$, and Michael C. Anderson ${ }^{2}$ \\ ${ }^{1}$ Institute of Psychology, Erasmus University Rotterdam, Rotterdam, The Netherlands \\ ${ }^{2} \mathrm{MRC}$ Cognition and Brain Sciences Unit, Cambridge, UK
}

\begin{abstract}
Research on retrieval suppression has produced varying results concerning whether negatively valenced memories are more or less suppressible than neutral memories. This variability may arise if, across studies, participants adopt different approaches to memory control. Cognitive and neurobiological research points to two mechanisms that achieve retrieval suppression: thought-substitution and direct suppression (Benoit \& Anderson, 2012; Bergström, de Fockert, \& Richardson-Klavehn, 2009). Using the Think/No-think paradigm, this study examined whether participants can inhibit neutral and negatively valenced memories, using a uniform direct suppression strategy. Importantly, when strategy was controlled, negative and neutral items were comparably inhibited. Participants reported high compliance with direct suppression instructions, and success at controlling awareness predicted forgetting. These findings provide the first evidence that direct suppression can impair negatively valenced events, and suggest that variability in forgetting negative memories in prior studies is unlikely to arise from difficulty using direct suppression to control emotionally negative experiences.
\end{abstract}

Keywords: Memory control; Direct suppression; Think/No-think paradigm.

For better or for worse, people regularly encounter reminders of things past. Objects, people, or situations can revive memories of a birthday celebration or a meeting with a friend, but can also remind us of the loss of a loved one. When negative memories intrude, they unsettle us, undermining our peace of mind, and people generally take mental action to limit the duration of such memories in awareness. Studying the mechanisms underlying this type of memory control and the limits on their operation is fundamental to understanding how people adapt the functioning of their memories in the aftermath of unpleasant life experiences.

The ability to control unwanted memories has been studied with the Think/No-think (TNT) paradigm (Anderson \& Green, 2001). The TNT paradigm investigates how suppressing retrieval in

Correspondence should be addressed to: Kevin van Schie, Institute of Psychology, Erasmus University Rotterdam, PO Box 1738, NL-3000 DR Rotterdam, The Netherlands. E-mail: k.vanschie@fsw.eur.nl 
response to reminders affects a memory's retention. Participants first learn cue-target word pairs (e.g., ordeal-roach). They then perform a TNT task in which they receive trials presenting a cue from these pairs, and are asked to either recall the associated memory (i.e., think items), or to suppress its retrieval (no-think items). A third set of items (baseline items) is also studied, but is not shown in the TNT phase. Afterwards, memory for all pairs is assessed.

On the final test, a counterintuitive effect arises revealing the consequences of suppressing retrieval: no-think items are also recalled more poorly than baseline items that were neither retrieved nor suppressed. This difference, known as the negative control effect, shows that retrieval suppression causes more forgetting than would ordinarily occur due to the passage of time, and has been found in numerous studies (Anderson \& Green, 2001; Anderson et al., 2004; Anderson, Reinzholz, Kuhl, \& Mayr, 2011; Paz-Alonso, Ghetti, Matlen, Anderson, \& Bunge, 2009; see also Anderson \& Huddleston, 2011; Levy \& Anderson, 2008, for reviews). The negative control effect generalises to non-verbal materials, such as faces (Depue, Banich, \& Curran, 2006; Hanslmayr, Leipold, \& Bäuml, 2010; Hanslmayr, Leipold, Pastötter, \& Bäuml, 2009) and scenes (Depue et al., 2006; Depue, Curran, \& Banich, 2007).

A key question, however, concerns how effectively suppression works for emotional memories. The literature contains diverging views on how emotion might influence memory suppression. On the one hand, suppressing emotional memories might be intrinsically more difficult because these memories are better encoded, consolidated and retrieved, than are non-emotional ones (Hamann, 2001; LaBar \& Cabeza, 2006; Levine \& Pisarro, 2004). Memory enhancement for arousing emotional information also seems more automatic than it is for non-arousing information (Kensinger \& Corkin, 2004). Moreover, the intrusive nature of traumatic memories in posttraumatic distress disorder (PTSD) reinforces this view, suggesting that emotional memories are harder to suppress (Shipherd \& Beck, 2005). On the other hand, the emotionality of memories could motivate people to engage in cognitive control over emotional memories. Disruption of those memory traces might be easier precisely because emotional memories are more accessible (Levy \& Anderson, 2012).

Studies examining the ability to suppress retrieval of emotional memories produce evidence for both arguments. Some authors have found larger negative control effects for emotionally negative compared to neutrals or positive materials (e.g., Depue et al., 2006; Joorman, Hertel, Brozovich, \& Gotlib, 2005; Lambert, Good, \& Kirk, 2010), indicating people may be better able or more motivated to forget unpleasant stimuli. Others have found smaller effects for negative materials (e.g., Marx, Marshall, \& Castro, 2008; Nørby, Lange, \& Larsen, 2010). Others have found no measurable effects for valence (Murray, Muscatell, \& Kensinger, 2011). It thus remains unclear why the relative magnitude of negative control effects for neutral and negative memories has varied so much.

One possibility is that this variability stems from participants adopting different strategies for retrieval suppression across valence conditions and studies. One strategy that has received considerable interest is thought-substitution, which often produces larger negative control effects than are typically observed without specific strategy instructions (e.g., Hertel \& McDaniel, 2010; Joormann, Hertel, LeMoult, \& Gotlib, 2009). For example, Aided participants are often provided with alternative words (thought-substitutes) to retrieve when no-think cues appear, whereas Unaided participants received no specific guidance. The Aided group often show a larger negative control effect compared to the Unaided group.

If thought-substitution increases the negative control effect, uncontrolled variation in this strategy may account for variability in how effectively negative memories are forgotten. By this hypothesis, forgetting emotional memories is more difficult because inhibitory control is inadequate to handle the putative intrusiveness of negative traces. Thus, unless participants resort to thought-substitution, negative control effects 
for negative materials will be smaller. Greater negative control effects for negative memories could arise if negative memories prompted thoughtsubstitution more often than neutral memories, obscuring an underlying deficit in the ability to inhibit negative memories.

A second mechanism contributing to retrieval suppression is known as direct suppression (Levy \& Anderson, 2008). Direct suppression is assayed by asking participants to avoid thinking of the target memory without replacing it with anything else; if the unwanted memory happens to come to mind, participants simply are asked to block it out. Using direct suppression instructions, several studies have shown impaired recall for no-think items (Benoit \& Anderson, 2012; Bergström, de Fockert, \& Richardson-Klavehn, 2009; Hanslmayr et al., 2009). Importantly, both ERP and $\mathrm{fMRI}$ research comparing direct suppression with thought-substitution indicate that direct suppression is mediated by distinct control mechanisms, with the former suppressing neural processes that contribute to episodic retrieval (Benoit \& Anderson, 2012; Bergström et al., 2009; Hanslmayr et al., 2010). For instance, using effective connectivity methods, Benoit and Anderson (2012) demonstrated that direct suppression engages right dorsolateral prefrontal cortex to down-regulate hippocampal activity, but thought-substitution does not; thoughtsubstitution, by contrast, engages left ventrolateral prefrontal cortex to retrieve substitute memories, and is associated with increased retrieval-related activity in the hippocampus. Thus, these qualitatively different approaches to memory control are known to be dissociable at the neural level. Importantly, they indicate that inhibitory control can directly suppress retrieval of unwanted memories, impairing their retention. It remains unknown, however, whether direct suppression impairs negatively valenced memories, as all studies of direct suppression have used neutral items. Direct suppression may be especially ineffective in suppressing emotional content.

If inhibiting negative memories is difficult and thought-substitution is necessary to control such memories, then holding subjects' strategy constant by asking them to perform direct suppression should reveal less forgetting for negative compared to neutral memories. To examine this, we gave participants direct suppression instructions and encouraged them to use this approach, reducing variability in strategies. Additionally, we manipulated the emotional valence of the cue (neutral vs. negative) and of the target (neutral vs. negative). With this manipulation we could disentangle how direct suppression affects materials with different valences, and whether this effect is related to either the cue or target valence. The negative emotional valence of visible reminders may capture participants' attention, perhaps exaggerating any modulatory influence that affect may have on suppression (whether the influence is positive, or negative).

\section{METHOD}

\section{Participants}

Thirty-eight undergraduates $\left(M_{\text {age }}=20.63\right.$ years, $S D=2.03$, 10 males) of the Erasmus University Rotterdam participated for course credit. Participants were excluded if they had a diagnosis of attention deficit disorder, did not have Dutch as a first language (learned prior to age 5) or if they were colour blind. Two participants were not used in analyses because they had a score higher than 3 on three post-experimental non-compliance questions for no-think instructions.

\section{Materials and design}

The stimuli consisted of 72 weakly related Dutch cue-target word pairs (e.g., lane-meter, removecancer), 24 each in the think, no-think, and baseline conditions. Words and word pairs were in part selected from previous TNT studies (Anderson \& Green, 2001; Anderson et al., 2004) and in part newly constructed. Care was taken to ensure that cue and target words were only relatable to each other and not to items from other pairs. First through careful independent inspection by the experimenter and the first author, and then by ensuring that use of our 
materials was justified by inspection of the Dutch association norms.

Of the 24 pairs in each condition, valence (negative vs. neutral) of the cue and target words was manipulated, resulting in four valence groups: neutral-neutral, negative-neutral, neutral-negative, and negative-negative. Within each valence group six pairs each made up the think, no-think, and baseline conditions.

To assess valence and arousal, 25 students who did not participate in the experiment rated a large sample of words on valence ranging from 0 (Negative) to 50 (Positive) and arousal ranging from 0 (Low) to 50 (High). Our 72 words were selected from this sample. Words scoring under 16.7 on valence qualified as negative; those between 16.7 and 33.3 as neutral. For each valence group, valence (v), arousal (a), and word length (wl) of cue and target is reported: cue for neutralneutral pairs $\left(M_{\mathrm{v}}=26.65 ; M_{\mathrm{a}}=15.34, M_{\mathrm{wl}}=5.44\right)$, target for neutral-neutral pairs $\left(M_{\mathrm{v}}=27.88\right.$; $\left.M_{\mathrm{a}}=15.33, M_{\mathrm{wl}}=5.28\right)$; cue for neutral-negative pairs $\left(M_{\mathrm{v}}=27.9 ; M_{\mathrm{a}}=17.33, M_{\mathrm{wl}}=5.61\right)$, target for neutral-negative pairs $\left(M_{\mathrm{v}}=10.5 ; M_{\mathrm{a}}=24.41\right.$ $\left.M_{\mathrm{wl}}=7.28\right)$; cue for negative-neutral pairs $\left(M_{\mathrm{v}}=\right.$ 10.98; $\left.M_{\mathrm{a}}=23.75, M_{\mathrm{wl}}=5.83\right)$, target for negative neutral pairs $\left(M_{\mathrm{v}}=27.38 ; M_{\mathrm{a}}=17.64, M_{\mathrm{wl}}=\right.$ 5.28); cue for negative-negative pairs $\left(M_{\mathrm{v}}=\right.$ $\left.12.04 ; \quad M_{\mathrm{a}}=23.67, \quad M_{\mathrm{wl}}=6.72\right)$, target for negative-negative pairs $\left(M_{\mathrm{v}}=11.27 ; M_{\mathrm{a}}=23.61\right.$, $\left.M_{\mathrm{wl}}=6.28\right)$.

Within each valence group, critical pairs were counterbalanced so that each participated in every condition of the Think/No-think task equally often.

\section{Think/No-think procedure}

The experiment was run with E-prime 2.0 (Psychology Software Tools, Pittsburgh, PA), using a procedure based on Anderson et al.'s (2004) experiment. During the phases, the experimenter sat behind the participant, scoring vocal responses, giving participants instructions and verbally encouraging them when necessary.
Learning phase. Each pair appeared once individually in white font in the middle of a black screen for $5,000 \mathrm{~ms}$ ( $400 \mathrm{~ms}$ ITI). Pseudo-randomised test-feedback cycles followed in which participants responded with the target into a microphone when a cue appeared. Cues disappeared after 3,500 ms or when the participant responded. Regardless of the answer, the correct target appeared in green for $1,000 \mathrm{~ms}$ (400 ms ITI). As is standard in the TNT task test-feedback cycles continued until a participant acquired a minimum of $50 \%$ of target answers for all conditions combined. Participants had up to seven cycles to achieve the criterion.

Think/No-think phase. Participants were told that they would be receiving two types of trials in which a cue word from one of the pairs would appear for a short time. They were told that when the cues appeared in blue, they should immediately think of the associated target word and to keep it in mind while the cue was on screen. When the cues appeared in yellow, however, they were asked to stop themselves from thinking of the associated word. For these no-think trials, participants received direct suppression instructions, asking them to continuously focus on the cue and suppress retrieval of the target by blocking thoughts about it, without replacing it with other thoughts. Each trial started with a fixation cross $(400 \mathrm{~ms})$ followed by the cue $(3,500 \mathrm{~ms} ; 400 \mathrm{~ms}$ ITI). The colour representing each condition was counterbalanced over participants. The TNT phase took 40 minutes.

Participants performed 24 practice filler trials (12NT, 12T), followed by the experimental TNT phase. The TNT phase consisted of six blocks containing 96 think and no-think cues displayed in a pseudo-randomised order, with each think and no-think cue appearing twice; no more than three T or NT items appeared consecutively. Across all blocks, each think and no-think cue was repeated 12 times. Breaks of 30-45 seconds occurred between blocks.

Final test phase. All 72 pairs were tested with a "same probe" test (e.g., lane-___ Each cue 
appeared once in white font in the middle of a black screen for 3,500 ms (400 ms ITI). Participants were instructed to recall all words regardless of their colour and instruction in the TNT phase.

After the test, participants filled out a questionnaire in which they rated their success at controlling memory for each pair on a scale from 0 (Never able to avoid thinking about the target word) to 4 (Always able to avoid thinking about the target word). They also rated how often they used each of a collection of strategies on a scale from 0 (Never) to 4 (Always), and whether they intentionally were non-compliant with no-think instructions; 0 (Never) to 4 (Very frequently).

\section{RESULTS}

Analysis was based only on pairs for which participants recalled the target on the final learning test (Anderson et al., 2004). This moderately high level of learning performance yielded data in every cell for every participant. Analyses of variance (ANOVAs) were conducted to examine the after-effects of memory control in the final test. Counterbalancing condition was included as a between-subjects factor in all analyses to account for item effects, and nonsignificant results of this factor (or its interactions) are not reported. Instruction (think, no-think, and baseline) was analysed as a within-subjects factor. A multivariate test (Pillai-Bartlett trace; $V$ ), is reported when the assumption of sphericity was violated.

\section{Learning phase}

No participant required more than three cycles to achieve the learning criterion of $50 \%(M=1.69$, $S D=0.71)$. Overall, recall on the final learning test was $75.35 \%(S E=1.80)$, and was similar for words from different instruction conditions: baseline $(M=73.61, S E=2.15)$, think $(M=75.00$, $S E=2.32)$, no-think $(M=77.43, \quad S E=1.82)$, $F(2,70)=2.06, p>.05, \eta_{\mathrm{p}}^{2}=.06$. Recall for the valence conditions differed, $F(3,90)=18.41$, $p<.001, \eta_{\mathrm{p}}^{2}=.38$, with $M=77.62(S E=3.05)$,
$78.70(S E=1.99), 80.25(S E=1.82)$, and 62.81 $(S E=2.88)$ for neutral-neutral, neutral-negative, negative-neutral, and negative-negative, respectively. Paired sample $t$-tests (against Bonferronicorrected $\alpha$ of .0083) revealed that only the negative-negative condition differed from other groups, $t \mathrm{~s}(35)>5.2, p s<.001$. For these reasons, we conditioned our analysis of participants' final recall on having correctly learned items on the final learning test.

\section{Overall effects of memory control on the final test}

Instructions (baseline, no-think, think) affected recall, $V=0.25, F(2,29), p<.05, \eta_{\mathrm{p}}^{2}=.25$. The effect of instruction was followed up with two contrasts, comparing baseline to either the think or the no-think condition. Recall was poorer for words in the no-think condition $(M=0.82, S E=$ $0.04)$, compared to baseline $(M=0.91, S E=$ $0.02), F(1,33)=6.86, p<.05, \eta_{\mathrm{p}}^{2}=.17$. Conversely, recall for words in the think condition $(M=0.94, S E=0.01)$ was better than baseline, $F(1,33)=5.80, p<.05, \eta_{\mathrm{p}}^{2}=.14$. Thus, the present findings replicate previous reports (Benoit \& Anderson, 2012; Bergström et al., 2009; Hanslmayr et al., 2009, 2010) showing that direct suppression can impair recall of unwanted memories.

\section{Effects of cue valence}

To see whether cue valence influenced suppression success, Cue Valence (neutral, negative) was included as a within-subject factor. Here again, recall varied with instruction, $V=0.26, F(2,32)$, $p<.01, \eta_{\mathrm{p}}^{2}=.26$. Recall was lower for no-think words compared to baseline words, $F(1,33)=$ 4.96, $p<.05, \eta_{\mathrm{p}}^{2}=.13$, whereas recall was higher for think words than for baseline words, $F(1$, $33)=6.94, p<.05, \eta_{\mathrm{p}}^{2}=.17$. Overall recall did not vary with cue valence, $F<1$. Importantly, we observed no Cue Valence $\times$ Instruction interaction, $F<1$. Consequently, the contrast comparing baseline and no-think performance did not interact with cue valence, $F<1$, nor did the 

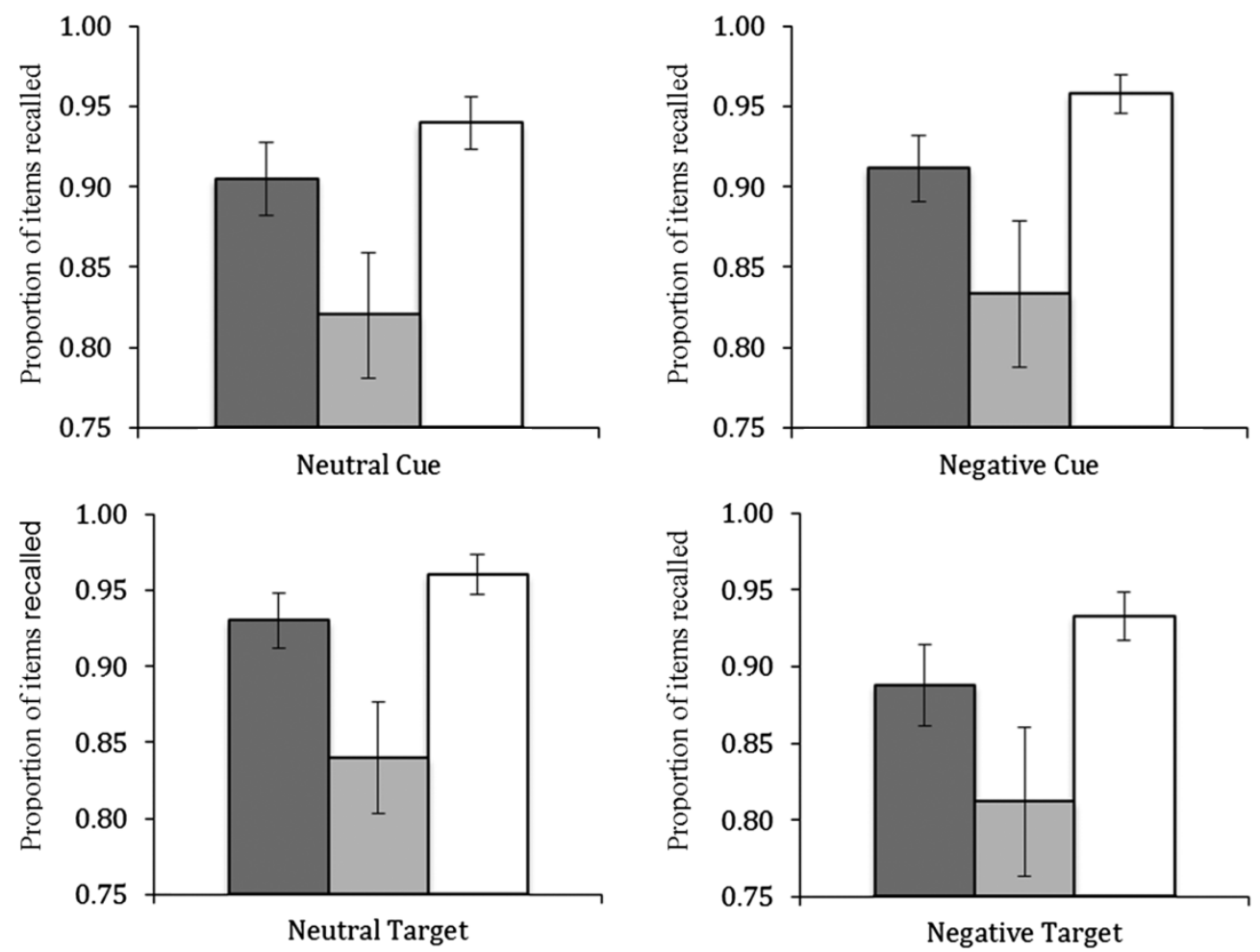

$\square$ Baseline $\square$ No-Think $\square$ Think

Figure 1. Mean proportion of recall for baseline (dark grey), no-think (light grey) and think (white) on the final test, displayed for valence of cue and target. Error bars represent standard errors of the mean.

contrast of baseline and think performance, $F<1$ (see Figure 1, top row, for the effects of instruction as a function of cue valence). Thus, when using a direct suppression strategy, people's ability to suppress unwanted memories did not vary for memories cued by negative or neutral reminders.

\section{Effects of target valence}

To see whether the valence of the to-besuppressed memories affected suppression success, Target Valence (neutral, negative) was added as a within-subjects factor. Again, recall varied with instruction, $V=0.26, F(2,32)=5.65, p<.01$, $\eta_{\mathrm{p}}^{2}=.26$. Contrasts revealed that baseline words were better recalled than no-think words,
$F(1,33)=4.96, \quad p<.05, \eta_{\mathrm{p}}^{2}=.13$, but were worse recalled than think words, $F(1,33)=$ 6.94, $p<.05, \eta_{\mathrm{p}}^{2}=.17$. Overall recall varied according to target valence, $F(1,33)=4.95$, $p<.05, \quad \eta_{\mathrm{p}}^{2}=.13$, with negatively valenced words being recalled less. Importantly, there was no interaction between Instruction and Target Valence, $F<1$ (see Figure 1, lower row, for the effects of instruction as a function of target valence). Thus, when using a direct suppression strategy, negative memories were neither more nor less suppressible than were neutral memories. These findings indicate that negatively valenced memories are capable of being forgotten by a direct suppression mechanism, and do not have to be forgotten by thought-substitution. 


\section{Questionnaire analysis}

Strategies for memory control. Most participants (88.89\%) frequently employed strategies during no-think trials that are consistent with direct suppression instructions, such as staring intently at the hint word, repeating the hint word silently, or letting their mind go blank in response to the hint word. Thought-substitution strategies in which people used the cue to generate an alternative word, thought or sound, were infrequently used (13.89\%). Thus, subjects largely followed our instructions to use direct suppression.

Self-reported success of memory control. Participants rated their success at controlling awareness for each of the suppression cues. We combined their ratings across items according to our distinctions: success at control for neutral versus negative cues, and success at control for neutral versus negative targets. Ratings for pairs were considered only if participants recalled the target word on the final trial in the learning phase, ensuring that differences in learning for different pair types did not contribute to success ratings. Participants reported greater success of not thinking of the memories associated to negative cues $(M=2.46, S E=0.11)$ compared to those associated to neutral cues $(M=2.30, S E=0.12), F(1,33)=5.94, p<.05$, $\eta_{\mathrm{p}}^{2}=.15$. For target valence, participants reported being more successful at not thinking of negative targets $(M=2.44, S E=0.12)$, compared to neutral targets $(M=2.32, S E=0.11)$, although this relationship was only marginal, $F(1,33)=3.41$, $p=.073, \eta_{\mathrm{p}}^{2}=.09$. These findings suggest, intriguingly, that, contrary to the notion that negative memories are more difficult to suppress, our participants experienced greater success at controlling negative memories.

Success ratings and memory inbibition. Interestingly, we found a relation between inhibition scores (baseline recall-no-think recall) and selfreported success at memory control. There was a relationship between success at controlling awareness and inhibition scores for negative cues, $r=$ $.42, p<.01$, and negative targets, $r=.37, p<.05$.
This relationship was not present for neutral cues or targets, $p>.05$. Thus, at least for negative memories, forgetting resulting from direct suppression was related to the participants' experience of success in controlling conscious awareness.

\section{DISCUSSION}

The present study examined whether unwanted memories could be forgotten with direct suppression, and compared the efficacy of this strategy for both neutral and negatively valenced memories. Consistent with recent work (Benoit \& Anderson, 2012; Bergström et al., 2009; Hanslmayr et al., 2009; Hanslmayr et al., 2010), negative control effects were indeed observed, illustrating how memory control can be accomplished without thought-substitution, via inhibitory processes that expel unwanted memories from awareness. This conclusion is supported by participants' high compliance with our instructions, and by correlations between the negative control effect and self-reports of control over awareness. Thus, thought-substitution need not be engaged to forget unwanted memories.

More importantly, however, the current study establishes, for the first time, that emotionally negative memories can be forgotten by direct suppression. All previous studies demonstrating negative control effects for emotional memories, used no particular suppression instructions (e.g., Lambert et al., 2010; Marx et al., 2008; Murray et al., 2011), and so it cannot be known what processes caused these effects. Moreover, all studies of direct suppression have used neutral materials, leaving it unclear whether negative memories can be controlled in this fashion. These findings do not support the idea that negatively valenced materials necessarily undermine people's ability to control memory. Nor do they clearly address whether they might enhance memory suppression. However, the simple verbal materials in our study may not capture the emotional qualities people experience in unpleasant life events. Therefore, more work is necessary to determine whether these effects generalise to 
materials that are more naturalistic and autobiographical and that might cause discomfort, shame, embarrassment, or sadness (see Noreen \& MacLeod, 2012).

Our findings speak to the potential causes of variability in the effect of valence on retrieval suppression (see Anderson \& Huddleston, 2011). Of interest was whether variations in the spontaneous use of thought-substitution in prior studies masked a deficit in inhibiting negative memories. Crucially, when we controlled strategy with direct suppression instructions, no inhibition deficit was evident; neutral and negative memories were comparably forgotten. Because the instructions used here are known to trigger top-down inhibitory modulation of hippocampal activity by dorsolateral prefrontal cortex (Benoit \& Anderson, 2012), our manipulation provided a theoretically refined test of the hypothesised difficulty in inhibiting negative memories. Indeed, to our surprise, participants judged pairs including negative cues or targets to have been easier to keep out of awareness than pairs with neutral cues or targets. Interestingly, success ratings predicted forgetting for negative, but not neutral memories, suggesting that controlling awareness of negative memories may be more reliant on inhibition, consistent with the possibility that they are more intrusive. Even with this possibility, however, our results as a whole, do not suggest that inhibition is less able to contend with negative materials. Nevertheless, uncontrolled variations in thoughtsubstitution may still explain variability in prior studies as to whether negative or neutral memories are more forgettable. Our findings simply show that this variability in thought-substitution, if it exists, does not mask a deficit in inhibiting negative memories.

Our experiment also manipulated both cue and target valence to examine which influences memory suppression. The majority of studies examining the negative control effect either show enhanced forgetting for neutral (Marx et al., 2008; Nørby et al., 2010) or for emotional materials (Depue et al., 2006; Joorman et al., 2005; Lambert et al., 2010). We observed neither effect, regardless of whether we considered cue or target valence. Nevertheless, these findings offer insights into several studies that show preferential suppression of emotional or non-emotional material. For instance, our study is the only one comparable to Lambert et al. (2010), who manipulated cue rather than target valence. Lambert et al. (2010) reported enhanced suppression for negative pairs, yet solely for neutral targets associated with a negative cue compared to neutral items paired with a positive cue. Because Lambert et al. (2010) lacked a condition with neutral cues, it was unclear whether these results reflected good forgetting of negative experiences, or difficulty suppressing positive items. Our finding of comparable forgetting for negative and neutral memories is compatible with theirs, but suggests that their finding may reflect diminished suppression of positive events and not enhanced suppression of negative ones.

Nørby et al. (2010) found greater forgetting for neutral than negative pairs, and suggested that this difference arose from the random intermixing of negative and neutral pairs. By this view, the difficulty in predicting emotional qualities of an upcoming item enhances the item's emotional salience, revealing how difficult it is to suppress negative memories. Studies in which predictability of emotional material is high, for instance through employing different blocks for emotional material (e.g., Depue et al., 2006, 2007), will show similar forgetting for negative and neutral items by this view, because the negative items are, in effect, less negative. Although we did not seek to address this issue, our findings suggest that random intermixing of valence types may not have the effects hypothesised by Nørby et al. (2010). In our study, negative and neutral items were intermixed and we nevertheless found emotional materials were comparably suppressible to non-emotional materials. Indeed, participants judged negative items to be easier to keep out of awareness. Clearly, our results are not in line with their account. Their finding of diminished negative control effects for negative materials thus remains unexplained.

One factor that may also contribute to variability in how suppression affects retention of negative and neutral memories concerns the 
semantic interrelatedness of the materials being suppressed. Because words of negative valence stem from a small set of categories (e.g., death, fear and violence) repeated retrieval of stimuli in the think condition (e.g., swamp-death) may affect later recall in the no-think condition (e.g., knife-murder) when there is high interrelatedness. Goodmon and Anderson (2011) have shown that high semantic relatedness in another inhibition phenomenon - retrieval-induced forgettingabolished forgetting effects. A similar principle may apply to retrieval suppression. The presence of uncontrolled inter-pair relationships may account for why Nørby et al. (2010) failed to observe negative control effects whereas we did, particularly given our efforts to control such relationships.

In conclusion, our results establish that negative memories may be forgotten via direct suppression and that thought-substitution is not essential (Benoit \& Anderson, 2012; Bergström et al., 2009; Hanslmayr et al., 2009). These findings imply that reports of forgetting of both negative and neutral memories in the TNT paradigm are unlikely to be due to deficient inhibitory control for negative memories that is compensated for by thought-substitution. Though we found that negative memories were inhibited, it remains possible that variability in the spontaneous use of thought-substitution occurs in other studies, potentially underpinning differences in the size of negative control effects across valence conditions. For these reasons, we recommend holding participants' strategic approach to the task constant to better understand the effects of emotion on the ability to suppress unwanted memories.

\section{Manuscript received 10 August 2012 Revised manuscript received 1 November 2012 Manuscript accepted 7 January 2013 First published online 6 February 2013}

\section{REFERENCES}

Anderson, M. C., \& Green, C. (2001). Suppressing unwanted memories by executive control. Nature, 410, 366-369. doi:10.1038/35066572
Anderson, M. C., \& Huddleston, E. (2011). Towards a cognitive and neurobiological model of motivated forgetting. In R. F. Belli (Ed.), True and false recovered memories: Toward a reconciliation of the debate. Nebraska Symposium on Motivation (Vol. 58, pp. 53-120). New York, NY: Springer. doi:10.1007/ 978-1-4614-1195-6_8

Anderson, M. C., Ochsner, K. N., Kuhl, B., Cooper, J., Robertson, E., Gabrieli, S. W., ... Gabrieli, J. D. E. (2004). Neural systems underlying the suppression of unwanted memories. Science, 303(5655), 232-235. doi:10.1126/science.1089504

Anderson, M. C., Reinzholz, J., Kuhl, B. A., \& Mayr, U. (2011). Intentional suppression of unwanted memories grows more difficult as we age. Psychology and Aging, 26(2), 397-405. doi:10.1037/a0022505

Benoit, R. G., \& Anderson, M. C. (2012). Opposing mechanisms support the voluntary forgetting of unwanted memories. Neuron, 76, 450-460. doi:10.1016/j.neuron.2012.07.025

Bergström, Z. M., de Fockert, J. W., \& RichardsonKlavehn, A. (2009). ERP and behavioural evidence for direct suppression of unwanted memories. NeuroImage, 48(4), 726-737. doi:10.1016/j.neuro image.2009.06.051

Depue, B. E., Banich, M. T., \& Curran, T. (2006). Suppression of emotional and nonemotional content in memory: Effects of repetition on cognitive control. Psychological Science, 17(5), 441-447. doi:10.1111/j.1467-9280.2006.01725.x

Depue, B. E., Curran, T., \& Banich, M. T. (2007). Prefrontal regions orchestrate suppression of emotional memories via a two-phase process. Science, 317(5835), 215-219. doi:10.1126/science. 1139560

Goodmon, L. B., \& Anderson, M. C. (2011). Semantic integration as a boundary condition on inhibitory processes in episodic retrieval. Journal of Experimental Psychology: Learning, Memory, and Cognition, 37(2), 416-436. doi:10.1037/a0021963

Hamann, S. (2001). Cognitive and neural mechanisms of emotional memory. Trends in Cognitive Sciences, 5, 394-400. doi:10.1016/S1364-6613(00)01707-1

Hanslmayr, S., Leipold, P., \& Bäuml, K.-H. (2010). Anticipation boosts forgetting of voluntarily suppressed memories. Memory, 18, 252-257. doi:10.10 80/09658210903476548

Hanslmayr, S., Leipold, P., Pastötter, B., \& Bäuml, K.-H. (2009). Anticipatory signatures of voluntary memory suppression. The Journal of Neuroscience, 29(9), 2742-2747. doi:10.1523/JNEUROSCI. 4703-08.2009 
Hertel, P., \& McDaniel, L. (2010). The suppressive power of positive thinking: Aiding suppressioninduced forgetting in repressive coping. Cognition and Emotion, 24(7), 1239-1249. doi:10.1080/ 02699930903172377

Joormann, J., Hertel, P. T., Brozovich, F., \& Gotlib, I. H. (2005). Remembering the good, forgetting the bad: Intentional forgetting of emotional material in depression. Journal of Abnormal Psychology, 114(4), 640-648. doi:10.1037/0021-843X.114.4.640

Joormann, J., Hertel, P. T., Lemoult, J., \& Gotlib, I. H. (2009). Training forgetting of negative material in depression. Journal of Abnormal Psychology, 118(1), 34-43. doi:10.1037/a0013794

Kensinger, E. A., \& Corkin, S. (2004). Two routes to emotional memory: Distinct neural processes for valence and arousal. Proceedings of the National Academy of Sciences, USA, 101, 3310-3315. doi:10.1073/pnas.0306408101

LaBar, K. S., \& Cabeza, R. (2006). Cognitive neuroscience of emotional memory. Nature Reviews Neuroscience, 7(4), 54-64. doi:10.1038/nrn1825

Lambert, A. J., Good, K. S., \& Kirk, I. J. (2010). Testing the repression hypothesis: Effects of emotional valence on memory suppression in the ThinkNo think task. Consciousness and Cognition, 19(1), 281-293. doi:10.1016/j.concog.2009.09.004

Levine, L. J., \& Pizarro, D. A. (2004). Emotion and memory research: A grumpy overview. Social Cognition, 22(5), 530-554. doi:10.1521/soco.22.5.530. 50767

Levy, B. J., \& Anderson, M. C. (2008). Individual differences in the suppression of unwanted memories: The executive deficit hypothesis. Acta
Psychologica, 127(3), 623-635. doi:10.1016/j. actpsy.2007.12.004

Levy, B. J., \& Anderson, M. C. (2012). Purging of memories from conscious awareness tracked in the human brain. Journal of Neuroscience, 32, 1678516794. doi:10.1523/JNEUROSCI.2640-12.2012

Marx, B. P., Marshall, P. J., \& Castro, F. (2008). The moderating effects of stimulus valence and arousal on memory suppression. Emotion, 8(2), 199-207. doi:10.1037/1528-3542.8.2.199

Murray, B. D., Muscatell, K. A., \& Kensinger, E. A. (2011). Effects of emotion and age on performance on a Think/No-think memory task. Psychology and Aging, 26, 940-955. doi:10.1037/a0023214

Nørby, S., Lange, M., \& Larsen, A. (2010). Forgetting to forget: On the duration of voluntary suppression of neutral and emotional memories. Acta Psychologica, 133(1), 73-80. doi:10.1016/j.actpsy.2009.10.002

Noreen, S., \& MacLeod, M. D. (2012). It's all in the detail: Intentional forgetting of autobiographical memories using the autobiographical Think/ No-think task. Journal of Experimental Psychology: Learning, Memory, and Cognition. Advance online publication. doi:10.1037/a0028888

Paz-Alonso, P. M., Ghetti, S., Matlen, B. J., Anderson, M. C., \& Bunge, S. A. (2009). Memory suppression is an active process that improves over childhood. Frontiers in Human Neuroscience, 3, 1-6. doi:10.3389/ neuro.09.024.2009

Shipherd, J. C., \& Beck, J. G. (2005). The role of thought suppression in posttraumatic stress disorder. Behavior Therapy, 36, 277-287. doi:10.1016/S00057894(05)80076-0 